\title{
Reliability-Aware Energy Optimization Algorithm for Network-on-Chip with Voltage-Scalable Links
}

\author{
Xiaona Xie ${ }^{1, *}$, Qingxin Zhu ${ }^{1}$ and Zhengwei Chang ${ }^{1,2}$ \\ ${ }^{1}$ School of Computer Science and Engineering, University of Electronic Science and Technology of China, Chengdu, Sichuan, 610054 , \\ P. R. China \\ ${ }^{2}$ Sichuan Electric Power Research Institute, Chengdu, Sichuan, 610072, P. R. China
}

Received: 22 Apr. 2013, Revised: 23 Aug. 2013, Accepted: 25 Aug. 2013

Published online: 1 Mar. 2014

\begin{abstract}
A reliability-aware energy optimization algorithm is proposed for Network-on-Chip (NoC) with voltage scalable links. Considering the effect of reduced voltage on fault rates, this approach achieves energy/reliability trade-off when performing routing path allocation and links voltage assignment statically. A novel energy-efficiency gradient driven heuristic is proposed to assign the voltages for the links during global design space exploration of routing path allocation. Experimental results show that the presented method can efficiently guarantee the communication reliability and bandwidth constraints, without significant loss of energy savings.
\end{abstract}

Keywords: Network-on-Chip, Reliability, Routing, Links, Voltage Assignment

\section{Introduction}

Network-on-Chip (NoC) [1] has been proposed to overcome the complex on-chip interconnect problem of SoCs in billion transistors. It has been shown that on-chip interconnects account for a significant fraction of the total energy consumption [2]. Regarding energy minimization, one promising technique for energy/performance trade-off of NoCs is to scale the speeds of the communication links with the corresponding voltage levels $[3,4]$.

Due to shrinking transistor geometries, smaller interconnect features, and higher operating frequencies, the occurrences of transient faults increase [5]. It has become clear that $100 \%$ reliable communication has to be relaxed in the presence of transient failures [6]. Thus, an important concern is to guarantee an imposed communication reliability goal under performance constraints, while minimizing the communication energy consumption [6].

Recent studies show that, voltage scaling, which is an efficient energy optimization technique, has a direct and negative effect on system reliability because of the increased rate of transient faults [7]. Consequently, it is especially challenging to address energy and reliability simultaneously. The existing voltage scaling algorithms in
NoCs achieve energy savings solely by scaling the communication tasks with respect to performance constraints $[8,9]$.

This paper proposes a design methodology for reliable and energy efficient NoCs. An approach to assign voltage levels for NoC links is presented, such that the communication reliability goal is satisfied and the communication energy consumption is minimized. At the same time, the performance of the resulting system is guaranteed through bandwidth reservation. In order to select optimal voltage levels, an efficient algorithm is presented to statically scale down the voltage and speed of links exploiting slack bandwidth for energy/reliability trader-offs. Experimental results show that the presented method can efficiently guarantee the communication reliability and bandwidth constraints, and obtain significant energy savings of NoC links.

\section{System models}

In this section, we introduce the notation used throughout this paper and present the system models.

\footnotetext{
*Corresponding author e-mail: xnxieok@163.com
} 


\subsection{NoC architecture}

In this work, we consider the hard NoC architecture with voltage-scalable links. According the network topology, there may be different routing paths between any two processing nodes. Each link is characterized with a set of discrete supply voltage values and the corresponding data transfer rates. The voltage level and speed of each link is assigned statically, based on the communication patterns and routing algorithms of target applications.

We denote a NoC platform with voltage-scalable links as a tuple $H N S=(C, A, L, P, V B)$, where $C=\left\{c_{1}, c_{2}, \ldots, c_{m}\right\}$ is the set of processing nodes, $A$ is the set of communication tasks, and each $a_{i j} \in A$ characterizes the communication task from $c_{i}$ to $c_{j}$. For every $a_{i j} \in A, u\left(a_{i j}\right)$ denotes the communication volume (bits) from $c_{i}$ to $c_{j}$, and $b\left(a_{i j}\right)$ denotes the bandwidth requirements (bits/second, $\mathrm{b} / \mathrm{s}$ ) that should be allocated. $L=\left\{l_{1}, l_{2}, \ldots, l_{n}\right\}$ is the set of links between nodes. $P=\left\{r p_{k}^{i j}|1 \leq i \leq| C|, 1 \leq j \leq| C \mid, k \geq 1\right\}$ is the set of all possible shortest routing path of the NoC, and the subset $R P^{i j}=\left\{r p_{1}^{i j}, r p_{2}^{i j}, \ldots, r p_{x}^{i j}\right\}$ is the set of routing paths from $c_{i}$ to $c_{j}$, and each $r p_{k}^{i j} \in R P^{i j}(1 \leq k \leq x)$ is the set of links along the $k$ th routing path from node $c_{i}$ to $c_{j}$. $V B=\left\{\left(V_{\min }, B_{\min }\right),\left(V_{1}, B_{1}\right),\left(V_{2}, B_{2}\right), \ldots\left(V_{\max }, B_{\max }\right),\right\}$ is the set of assignable link voltages and bandwidths (speeds). For convenience, $V L=\left\{V_{\text {min }}, V_{1}, V_{2}, \ldots, V_{\text {max }}\right\}$ is the set of scalable voltage levels of NoC links.

As shown in Fig. 1, an illustrative NoC architecture is composed of $3 \times 3$ nodes interconnected by a $2 \mathrm{D}$ mesh network. It consists of 9 processing nodes and 12 links, represented by $c_{1} \sim c_{9}$ and $l_{1} \sim l_{12}$. The communication task from $c_{1}$ to $c_{8}$ is represented by $a_{18}$, and the corresponding 3 shortest routing paths are $r p_{1}^{18}=\left\{l_{1}, l_{4}, l_{9}\right\}, \quad r p_{2}^{18}=\left\{l_{3}, l_{8}, l_{11}\right\} \quad$ and $r p_{3}^{18}=\left\{l_{3}, l_{6}, l_{9}\right\}$. The routing path $\left\{l_{3}, l_{8}, l_{11}\right\}$ is allocated for $a_{18}$. Another routing path $\left\{l_{7}, l_{5}\right\}$ is allocated for the communication task $a_{53}$.

For this NoC, The links voltages can be assigned $1 \mathrm{~V}$, $1.1 \mathrm{~V}$ or $1.2 \mathrm{~V}$, and the corresponding speeds are $0.83 \mathrm{G} \mathrm{b} / \mathrm{s}$, $0.92 \mathrm{G} \mathrm{b} / \mathrm{s}$ or $1 \mathrm{G} \mathrm{b} / \mathrm{s}$. For example, the speeds of $l_{7}$ and $l_{5}$ are assigned $0.92 \mathrm{G} \mathrm{b} / \mathrm{s}$ and $1 \mathrm{G} \mathrm{b} / \mathrm{s}$ respectively. As a result, the voltage level of the links is determined off-line, and will be fixed at runtime.

\subsection{Allocation/assignment model}

For a given $H N S$, an initial step allocates each communication task with a routing path, referred as routing path allocation in this paper. Each link is then assigned a voltage level and corresponding speed, referred as voltage assignment.

Formally, given $H N S=(C, A, L, P, V B)$, the allocation/assignment problem is to find the two mapping functions $\gamma: A \rightarrow P \Rightarrow r p_{k}^{i j}=\gamma\left(a_{i j}\right), \forall a_{i j} \in A, \exists r p_{k}^{i j} \in P$ and $\omega: L \rightarrow V L \Rightarrow V_{i}=\omega\left(l_{i}\right), \forall l_{i} \in L, \exists V_{i} \in V L$.

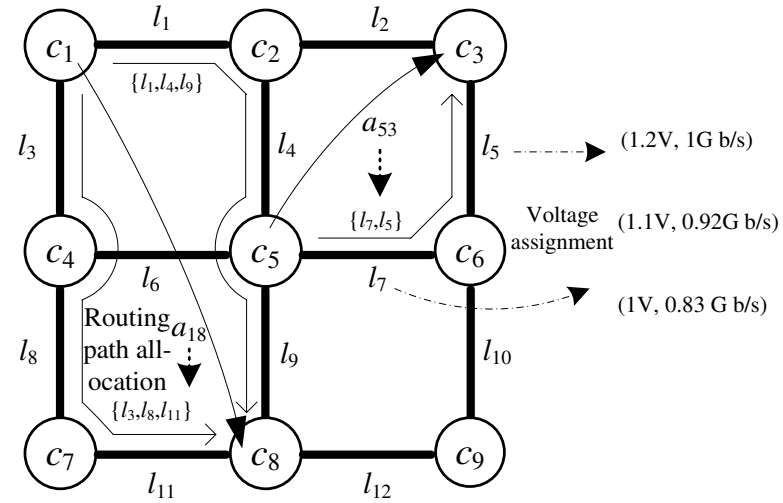

Fig. 1: NoC architecture and the illustration of the allocation/assignment problem.

Thus, the communication workload of link $l_{k}$ is:

$$
U_{k}(\gamma)=\sum_{\forall a_{i j} \in A \wedge l_{k} \in \gamma\left(a_{i j}\right)} u\left(a_{i j}\right)
$$

The reserved bandwidth of link $l_{k}$ is:

$$
B R_{k}(\gamma)=\sum_{\forall a_{i j} \in A \wedge l_{k} \in \gamma\left(a_{i j}\right)} b\left(a_{i j}\right) .
$$

\subsection{Energy model}

We consider the dynamic energy consumption caused by the communication tasks in a NoC. When scaling down the voltage of links to reduce energy, the clock frequency scales down, so does the transmission speed.

The energy consumed by a link per bit is modelled as:

$$
E_{L_{b i t}}=\frac{1}{2} C_{l} V_{d d}^{2}
$$

where $C_{l}$ is the load capacitance, and $V_{d d}$ is the supply voltage of the link.

The energy consumption of a link $l_{k}$ is:

$$
E_{k}(\gamma, \omega)=\frac{1}{2} C_{l_{k}} \omega\left(l_{k}\right)^{2} U_{k}(\gamma) .
$$

The total energy consumption of all the NoC links is:

$$
E(\gamma, \omega)=\frac{1}{2} \sum_{\forall l_{k} \in L} C_{l_{k}} \omega\left(l_{k}\right)^{2} U_{k}(\gamma)
$$

\subsection{Reliability model}

During the execution of a NoC-based embedded application, faults may occur due to various reasons, such as the effects of cosmic ray radiation or electromagnetic 
interference [10]. Since the transient faults are the most common, we focus on transient faults in this paper. Without loss of generality, it is assumed that the processing nodes and routers have been designed to achieve resilience from transient faults, so only the reliability of links must be considered.

Due to the occurrences of transient faults, each link of the NoC has two states: operational or failed. If a link fails during an idle cycle, since there is no packet to transport, the reliability of this link would not affect by this transient failure. We assume that the failure of a link follows a Poisson process with a fault rate $\lambda$, and failures of links are statistically independent. $\lambda$ describes the amount of faults that will occur per second. The reliability $R_{k}$ of a link $l_{k}$ is defined as the probability of its successful performability [11]. $l_{k}$ is:

With routing path allocation $\gamma$, the reliability of a link

$$
R_{k}(\gamma)=\exp \left(-\lambda \frac{U_{k}(\gamma)}{B_{\max }}\right)
$$

The communication reliability of the NoC is defined as the probability that communication tasks $A$ can transport successfully on the network links:

$$
R(\gamma)=\prod_{\forall l_{k} \in L} R_{k}(\gamma)
$$

\subsection{Energy/Reliability Model}

The equations presented so far do not account for the effects of voltage on reliability. However, lowering the voltage has been shown to dramatically lower the reliability [7]. Thus, the fault rate $\lambda$ is dependent on the voltage level that link $l_{k}$ is run at. The relation between the two can be expressed as [7]:

$$
\lambda\left(\omega\left(l_{k}\right)\right)=\lambda^{0} 10^{\frac{d\left(V_{\max }-\omega\left(l_{k}\right)\right)}{V_{\max }-V_{\min }}} .
$$

where $\lambda^{0}$ is the fault rate of link $l_{k}$ when run at maximum voltage $V_{\max }$, and $d$ is an architecture specific constant. Since the voltage level of the links can be changed between $V_{\max }$ and $V_{\text {min }}$, the fault rate at $V_{\min }$ is $10^{d}$ higher than fault rate at $V_{\max }$.

Let us now return to the communication reliability derived previously. Thus, the reliability of a link $l_{k}$ is:

$$
R_{k}(\gamma, \omega)=\exp \left(-\lambda\left(\omega\left(l_{k}\right)\right) \frac{U_{k}(\gamma)}{B\left(\omega\left(l_{k}\right)\right)}\right) .
$$

where $B\left(\omega\left(l_{k}\right)\right)$ is the bandwidth (speed) of link $l_{k}$.

The communication reliability of the NoC is:

$$
R(\gamma, \omega)=\exp \left(-\sum_{\forall l_{k} \in L} \lambda\left(\omega\left(l_{k}\right)\right) \frac{U_{k}(\gamma)}{B\left(\omega\left(l_{k}\right)\right)}\right)
$$

\section{Problem formulation}

The problem of energy-aware routing path allocation and links voltage assignment for NoC under reliability and performance constraints, which consists of two sub-problems, can be formulated as follows.

Input:

(1)The NoC model $H N S=(C, A, L, P, V B)$.

(2)The energy model.

(3)The reliability model: $\lambda^{0}, d$, and the reliability goal $R_{g}$.

\section{Output:}

The output of the problem consists of the routing path allocation $\gamma: A \rightarrow P$ and voltage assignment $\omega: L \rightarrow V L$, such that the total communication energy $E(\gamma, \omega)$ is minimized.

Constraints:

The communication reliability and performance constraints are satisfied:

$$
\begin{gathered}
R(\gamma, \omega) \geq R_{g} . \\
\forall l_{k} \in L, B\left(\omega\left(l_{k}\right)\right) \geq B R_{k}(\gamma) .
\end{gathered}
$$

where equation (11) guarantees that the communication reliability should be no less than the specified reliability goal. Equation (12) specifies the communication performance constraints for the problem in terms of the aggregated bandwidth requirements for each link. The allocation/assignment solution has to guarantee that the communication traffic (workload) of any link does not exceed the available bandwidth, such that the performance requirements between each communicating nodes pair can be satisfied.

\section{Reliability-aware links voltage assignment}

The allocation/assignment of NoC includes solving the following two sub-problems: routing path allocation and links voltage assignment. Finding an optimal mapping for NoC that consumes the least energy is known to be NP-hard [12]. Finding the optimum energy solution for the NoC architecture with voltage-scalable links is an even harder problem.

As Fig. 2 shown, we propose a design framework based on tabu search. During the tabu search based exploration of the design space, once we get a routing path configuration, we need to generate the optimal voltage assignment in order to calculate the fitness (object function) of the corresponding solution. The aim of this section is to introduce a new voltage assignment algorithm capable of identifying refined scaling voltages by considering the individual reliability/energy trade-off for links based on the allocated communication tasks.

We denote $\omega^{\prime}$ as the voltage assignment function when we scale down the voltage of link $l_{k}$ by a voltage 


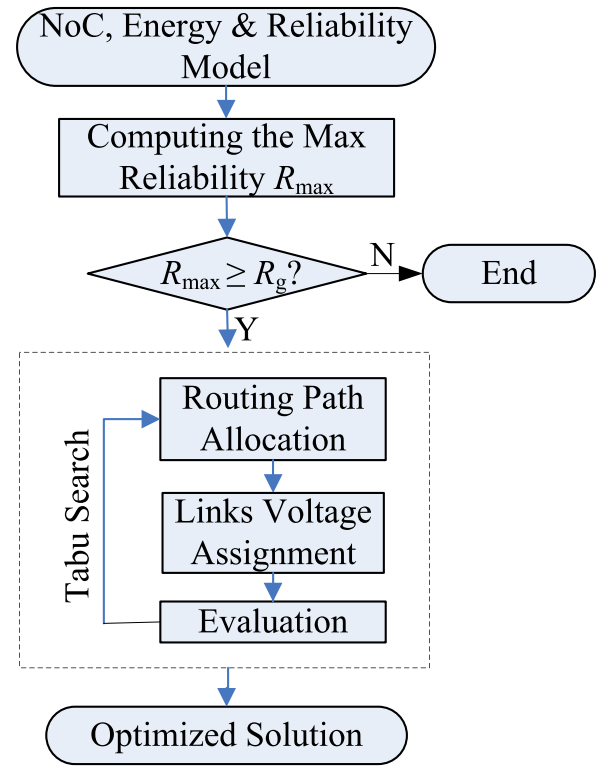

Fig. 2: Reliability-aware links voltage assignment.

quantum $\Delta v$, i.e. $\omega^{\prime}\left(l_{k}\right)=\omega\left(l_{k}\right)-\Delta v, \quad \forall l_{j} \neq l_{k}$, $\omega^{\prime}\left(l_{j}\right)=\omega\left(l_{j}\right)$. To demonstrate the principle behind our algorithm, the following definitions need to be given:

Definition 1. An energy gradient $\Delta E_{k}$ is defined as the difference between the communication energy consumption with the voltage assignment $\omega$ and the reduced energy consumption (due to voltage and frequency scaling of link $l_{k}$ ) of the NoC when $\omega\left(l_{k}\right)$ scaled down by $\Delta v$. Formally:

$$
\begin{aligned}
\Delta E_{k}(\omega) & =E(\omega)-E\left(\omega^{\prime}\right) \\
& =\frac{1}{2} C_{l_{k}}\left(\left(\omega\left(l_{k}\right)\right)^{2}-\left(\omega\left(l_{k}\right)-\Delta v\right)^{2}\right) U_{k}(\gamma) .
\end{aligned}
$$

Definition 2. A reliability gradient $\Delta R_{k}$ is defined as the difference between the communication reliability with the voltage assignment $\omega$ and the lowered reliability (due to voltage and frequency scaling of link $l_{k}$ ) of the NoC when $\omega\left(l_{k}\right)$ scaled down by $\Delta v$. Formally:

$$
\Delta R_{k}(\omega)=R(\omega)-R\left(\omega^{\prime}\right)=R(\omega) \times\left(1-\frac{R_{k}\left(\omega^{\prime}\right)}{R_{k}(\omega)}\right)
$$

where

$$
\begin{aligned}
& \frac{R_{k}\left(\omega^{\prime}\right)}{R_{k}(\omega)} \\
& =\exp \left[U_{k}(\gamma) \times \lambda_{k}^{0}\left(\frac{10^{\frac{d\left(V_{\max }-\omega\left(l_{k}\right)\right)}{V_{\max } V_{\min }}}}{B\left(\omega\left(l_{k}\right)\right)}-\frac{10^{\frac{d\left(V_{\max }-\omega\left(l_{k}\right)+\Delta v\right)}{V_{\max }-V_{\min }}}}{B\left(\omega\left(l_{k}\right)-\Delta v\right)}\right)\right]
\end{aligned}
$$

Definition 3. An energy-efficiency gradient $\Delta E E_{k}$ is defined as the ratio of energy gradient to reliability gradient of link $l_{k}$ when $\omega\left(l_{k}\right)$ scaled down by $\Delta v$. Formally:

$$
\Delta E E_{k}(\omega)=\Delta E_{k}(\omega) / \Delta R_{k}(\omega) .
$$

We use an energy-efficiency gradient driven heuristic to assign the voltage level for the links meeting the communication reliability and bandwidth constraints while considering the effects of voltage scaling on reliability. To efficiently minimize communications energy of the links, this algorithm chooses the most appropriate link in which the voltage level will be decreased by $\Delta v$ iteratively. Specifically, it is desirable to give higher priorities to links with higher energy gradient and lower reliability gradient. Consequently, the algorithm intentionally selects the best candidate link with the highest energy-efficiency gradient. To simplify the implementations, we define the following energy-reliability ratio function $\theta_{k}$ :

$$
\theta_{k}(\omega)=\Delta E E_{k}(\omega) \times R(\omega)=\frac{\Delta E_{k}(\omega)}{1-R_{k}\left(\omega^{\prime}\right) / R_{k}(\omega)} .
$$

Therefore, the best candidate $l_{k^{\prime}}$ can be chosen based on $\theta_{k^{\prime}}=\max \left\{\theta_{k} \mid l_{k} \in L\right\}$. The reason for using $\theta_{k}$ instead of $\Delta E E_{k}$ is that it just needs parameters of the only link to calculate $\theta_{k}$.

The energy-efficiency gradient driven links voltage assignment algorithm is expressed in the following steps.

//Input: the routing path allocation $\gamma$

$/ / Q_{E E}:$ the queue of links in decreasing order of $\theta_{k}$

(1)Initialize the voltage of each link, set $\omega\left(l_{k}\right)=V_{\max }$ for $\forall l_{k} \in L$. Set $R(\omega)=R(\gamma, \omega)$.

(2)For each $l_{k} \in L$.

(2.1)If $U_{k}(\gamma)>0$ and $B\left(\omega\left(l_{k}\right)-\Delta v\right) \geq B R_{k}(\gamma)$.

(2.1.1)Calculate $\Delta R_{k}, \Delta E_{k}$ and $\theta_{k}$.

(2.1.2)If $R(\omega)-\Delta R_{k}(\omega) \geq R_{g}$, insert $l_{k}$ into $Q_{E E}$.

(3)If $\left|Q_{E E}\right|>0$.

(3.1)Delete the first link, $l_{k}$, from $Q_{E E}$.

(3.2)Set $R(\omega)=R(\omega)-\Delta R_{k}(\omega), \quad$ and $\omega\left(l_{k}\right)=\omega\left(l_{k}\right)-\Delta v$.

(3.3)If $\omega\left(l_{k}\right)-\Delta v \geq V_{\text {min }}$ and $B\left(\omega\left(l_{k}\right)-\Delta v\right) \geq B R_{k}(\gamma)$ (3.3.1)Calculate $\Delta R_{k}, \Delta E_{k}$ and $\theta_{k}$.

(3.3.2)If $R(\omega)-\Delta R_{k}(\omega) \geq R_{g}$, insert $l_{k}$ into $Q_{E E}$.

(4)Repeat step 3 until $\left|Q_{E E}\right|==0$.

(5)Return $\omega$.

Obviously, the proposed algorithm aims at minimizing communication energy under two conditions: 1) lowering voltage levels will not result in missing bandwidth reservations, and 2) reliability constraints are satisfied. Based on these observations, the non idle links with the highest value of $\theta_{k}$ are iteratively scaled down until no further scaling is possible due to reliability and performance constraints. 
In some cases, there may be some available bandwidth unexploited due to the scaling granularity determined by the value of $\Delta v$. However, tabu search will try to obtain a better solution by explore the design space, such that the voltage assignment algorithm can reduce the energy consumption efficiently.

\section{Experimental Results}

The proposed algorithm was tested on a benchmark example to demonstrate its capability to produce high quality solutions in terms of energy and reliability. The system parameters used in our experiments are: $\lambda^{0}=10^{-7}$ and $\Delta v=0.1$. Table 1 gives the voltage and corresponding speed values of the NoC links.

Table 1: System parameters.

\begin{tabular}{cc}
\hline Voltage $(\mathrm{V})$ & Speed $(\mathrm{Gb} / \mathrm{s})$ \\
\hline 1.0 & 0.67 \\
1.1 & 0.73 \\
1.2 & 0.80 \\
1.3 & 0.86 \\
1.4 & 0.93 \\
1.5 & 1.00 \\
\hline
\end{tabular}

In the experiments, we consider 3 different schemes:

(1)RCEO (Reliability-aware Communication Energy Optimization): This is our proposed approach.

(2)CEO (Communication Energy Optimization): We applied the allocation/assignment approach in [8] with the objective of minimizing the energy consumption, but without imposing any reliability constraints.

(3)CEO+: CEO plus reliability constraints.

We experimented with a multimedia system with an H.263 encoder/decoder and an MP3 encoder/decoder [12]. This application of 25 nodes was mapped onto a $5 \times 52 \mathrm{D}$ mesh NoC, and $R_{\max }=0.9999999993$.

\subsection{Comparison of energy/reliability trade-off}

For the evaluation of our approach, we vary the value of $d$ (as 0, 2, and 4 respectively) and $R_{g}(0.999 \sim 0.999999$ 999). Fig. 3 and Fig. 4 present experimental results that compare RCEO with CEO and $\mathrm{CEO}+$. Because the reliability of $\mathrm{CEO}+$ solution is close to RCEO, the reliability of CEO+ is omitted for brevity.

As Fig. 3 and Fig. 4 shown:

(1)While the value of $d$ is fixed, the solutions of all 3 algorithms are roughly the same for lower reliability constraints $\left(R_{g}<0.9999\right)$. As $R_{g}$ increases, the results of CEO remain unchanged since it does not

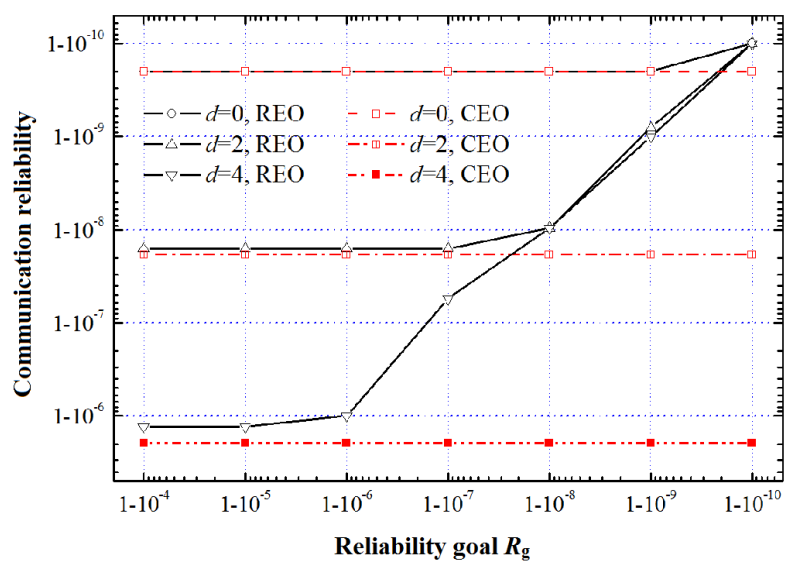

Fig. 3: Reliability solutions of different parameters.

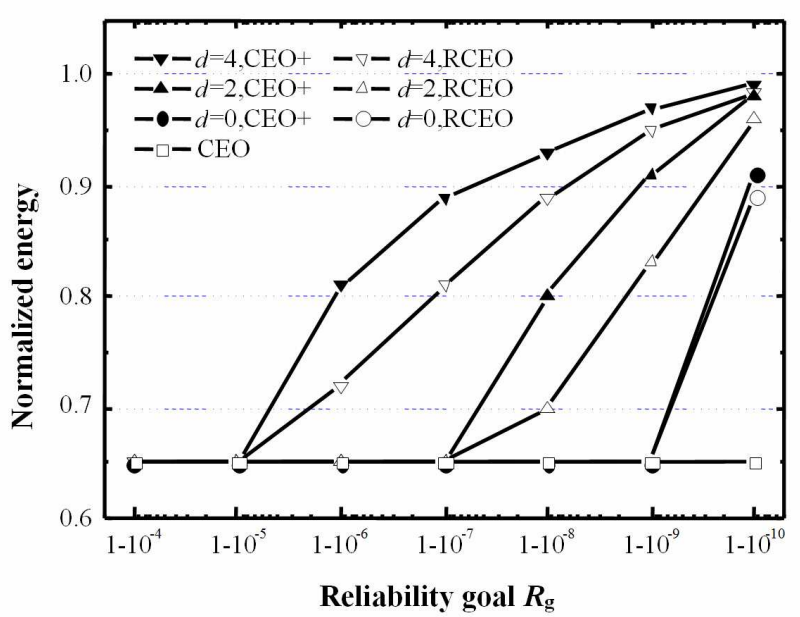

Fig. 4: Energy solutions of different parameters.

consider the reliability constraints, and the communication energy of RCEO and $\mathrm{CEO}+$ also increase to satisfy the reliability constraints.

(2)While the value of $d$ increases, the reliability of CEO decreased exponentially. Even when $d=0$ (i.e., constant fault rates), CEO results in lower reliability of 0.999999998 which comes from the extended transmission of messages due to lowered voltage and speed. Though the reliability goal is satisfied, CEO+ saves limited energy. In contrast, the solutions of RCEO try to reduce the energy consumption efficiently while guaranteeing the reliability goals with different values of $R_{g}$ and $d$.

For example, for $d=2$ and $R_{g}=0.9999999$, the unconstrained-reliability allocation/assignment solution determined by CEO saves $35 \%$ energy. But the reliability goal is missed, since the resulted reliability is only 0.999 999 815. However, by using RCEO we have made the 
designed system meet its reliability goal, sacrificing only $5 \%$ of the energy savings compared with CEO. On the same time, CEO+ only saves $20 \%$ energy.

\subsection{Results of links voltage assignment}

For $d=2$ and $R_{g}=0.9999999$, the links voltage assignment results of RCEO, CEO and CEO+ are counted to verify the principle of each algorithm.

Firstly, the numbers of NoC links with the same supply voltage are summarized, as shown in Fig. 5.

Then the communication workloads of the links with the same voltage are summarized, as shown in Fig. 6. Some of the links of $1.5 \mathrm{~V}$ are idle, i.e. without communication traffic.

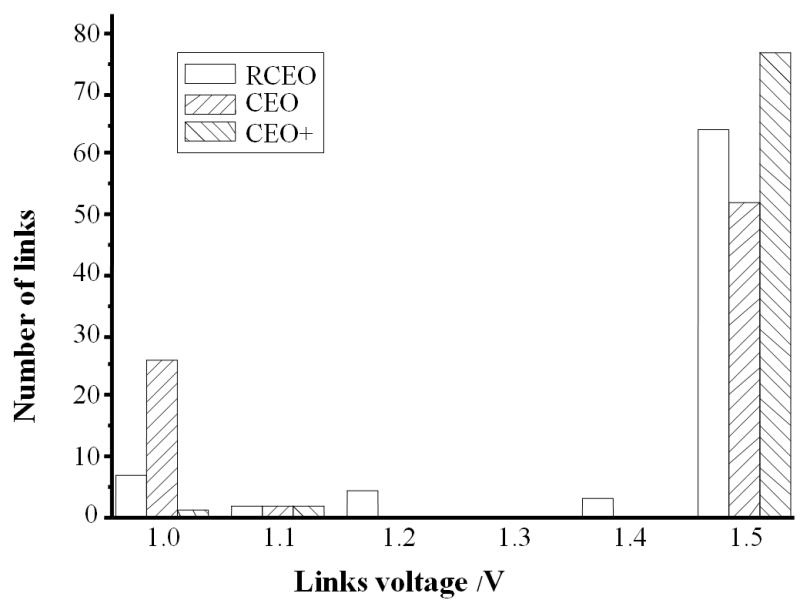

Fig. 5: The summarized number of links with the same voltage.

As Fig. 5 and Fig. 6 shown:

(1)CEO uses energy gradient driven links voltage assignment heuristic to decrease the voltage of links with heavy communication workloads firstly. The voltages of these links are decreased step by step under the bandwidth constraints. As a result, the number of links assigned $1.0 \mathrm{~V}$ are the most compared to RCEO and CEO+, and the communication workload of these links with the lowest voltage are the heaviest too. Moreover, the numbers of links of $1.5 \mathrm{~V}$ are the least. Thus CEO achieves the best energy savings with the poorest reliability.

(2)CEO+ uses energy gradient driven links voltage assignment heuristic considering reliability constraints. The decreasing voltage of links with heavier communication workload results to poor reliability, especially when the voltages of these

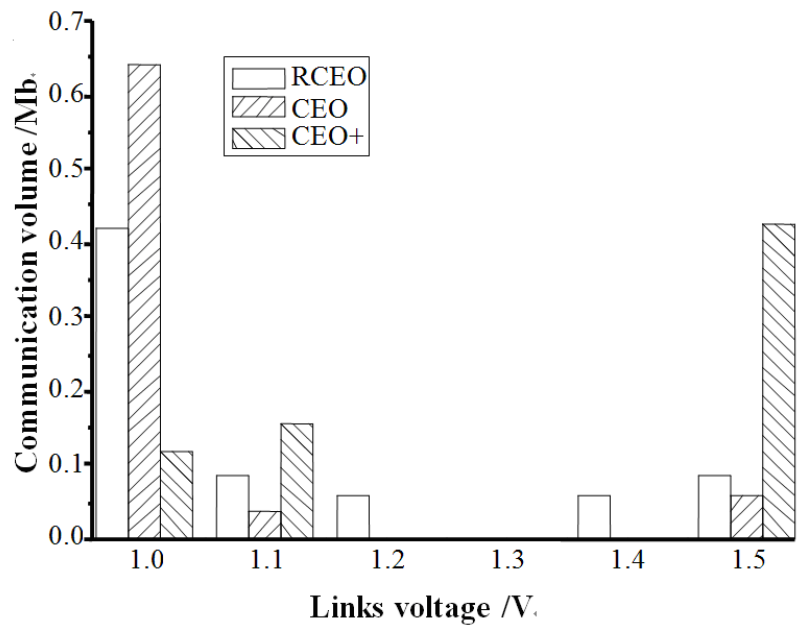

Fig. 6: The summarized communication volume of links with the same voltage.

links are close to $V_{\min }$. As a result, when some links with heavier communication workload have been decreased to $1.0 \mathrm{~V}$ or $1.1 \mathrm{~V}$, the $\mathrm{NoC}$ reliability has close to $R_{g}$, and the voltage assignment procedure has to ends. On the other way, most of the links remain $V_{\text {max }}=1.5 \mathrm{~V}$.

(3)Unlike CEO and CEO+, RCEO uses energy-efficiency gradient driven links voltage assignment heuristic to achieve energy and reliability trade-off. As a result, the communication workload of links with different voltages is more uniform.

\section{Conclusions}

Reliability is increasingly becoming an important issue in the design of embedded systems. However, existing energy optimization techniques for NoCs do not consider reliability requirements. We proposed an approach that took reliability into account when performing routing path allocation and links voltage assignment. Our approach is able to produce energy-efficient implementations which guarantee the performance and reliability requirements. As the experimental results shown, if the voltage level of NoC link was lowered to reduce energy consumption, the communication reliability was significantly reduced. By carefully deciding the links voltage assignment we can eliminate the negative impact of voltage scaling on NoC communication reliability without significant loss of energy savings.

\section{Acknowledgements}

This work was supported by the Natural Science Foundation of China (No. 61003032, 61100118), and 
Artificial Intelligence Key Laboratory of Sichuan Province of China (No. 2010RY010, 2011RYJ05).

\section{References}

[1] T. Bjerregaard and S. Mahadevan, A survey of research and practices of Network-on-chip. ACM Computing Surveys, 38, 1-51 (2006)

[2] F. Worm, P. Ienne, P. Thiran, and G. A. d. m. G. de micheli, An adaptive low-power transmission scheme for on-chip networks. In Proc. of the 15th International Symposium on System Synthesis, 92-100 (2002).

[3] J. Kim and M. A. Horowitz, Adaptive supply serial links with sub-1-V operation and per-pin clock recovery. IEEE Journal of Solid-State Circuits, 37, 1403-1413 (2002).

[4] S. Chu, S. Chen and S. Weng, CPPM: a comprehensive power-aware processor manager for a multicore system. Applied Mathematics and Information Sciences, 7, 793-800 (2013).

[5] C. Constantinescu, Trends and challenges in VLSI circuit reliability . IEEE Micro, 23, 14-19 (2003).

[6] S. Manolache, P. Eles, and Z. Peng, Fault-aware communication mapping for NoCs with guaranteed latency . International Journal of Parallel Programming, 35, 125-156 (2007).

[7] Z. Dakai, R. Melhem, and D. Mosse, The effects of energy management on reliability in real-time embedded systems . In Proc. of the International Conference on Computer Aided Design, 35-40 (2004).

[8] D. Shin and J. Kim, Power-aware communication optimization for networks-on-chips with voltage scalable links. In Proc. of the 2nd IEEE/ACM/IFIP International Conference on Hardware/software Codesign and System Synthesis, 170-175 (2004).

[9] G. Chen, F. Li, M. Kandemir, and M. J. Irwin, Reducing NoC energy consumption through compilerdirected channel voltage scaling. In Proc. of the ACM SIGPLAN Conference on Programming Language Design and Implementation, 193-203 (2006).

[10] T. Dumitras and R. Marculescu, On-Chip stochastic communication . In Proc. of the Conference on Design, Automation and Test in Europe, (2003).

[11] S. M. Shatz, J. P. Wang, and M. Goto, Task allocation for maximizing reliability of distributed computer systems . IEEE Transactions on Computers, 41, 1156-1168 (1992).

[12] J. Hu and R. Marculescu, Energy-aware mapping for tilebased NoC architectures under performance constraints In Proc. of the Conference on Asia South Pacific design automation, 233-239 (2003).
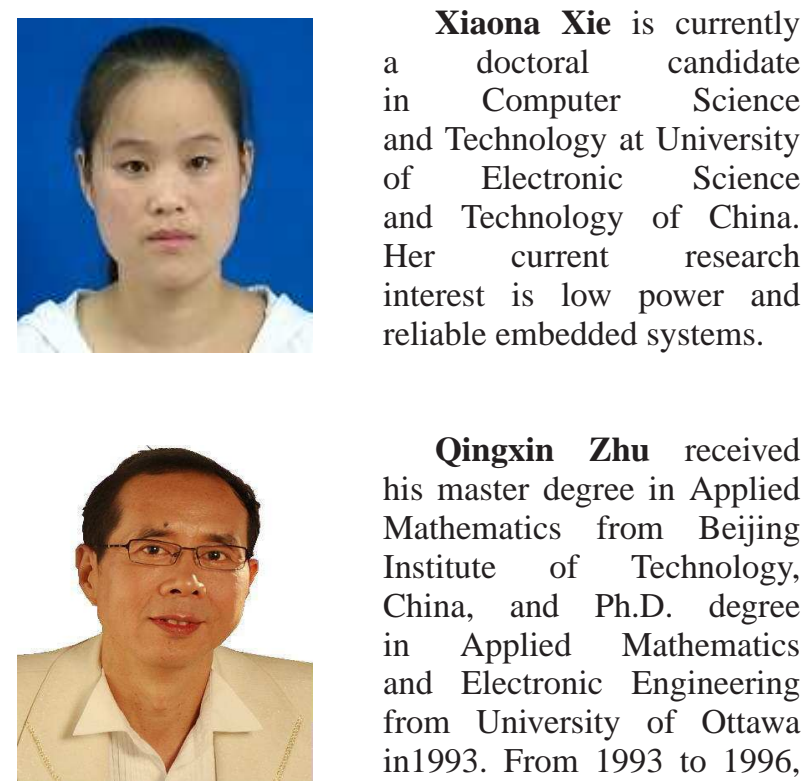

Qingxin Zhu received his master degree in Applied Mathematics from Beijing Institute of Technology, China, and Ph.D. degree in Applied Mathematics and Electronic Engineering from University of Ottawa in1993. From 1993 to 1996 , he pursued his postdoctoral research in electronic engineering at University of Ottawa, and in computer science at Carleton University, respectively. From 1996 to 1997, he was a senior researcher at Northern Telecom in Canada. From 1998, he was a professor at the Department of Computer Science and Engineering, University of Electronic Science and Technology of China, and is a doctoral supervisor from 2003 to present in the same department. From 2002 to 2003, he was a senior academic visitor in pattern recognition and computer vision at Concordia University, Canada. He is the director of the academic committee and the director of multimedia and virtual reality laboratory in the School of Computer Science and Engineering, University of Electronic Science and Technology of China. He is a senior member of Computer Academy of China. $\mathrm{He}$ is the director of multimedia committee of Sichuan, China. His research interests include computer vision, computer graphics, video processing, image processing, information security, optimal search, and optimal control theory. He published over 30 papers and 3 monographs; one of his monographs won the honor of outstanding academic composition series of China. He is a member of American Mathematics Society (AMS).

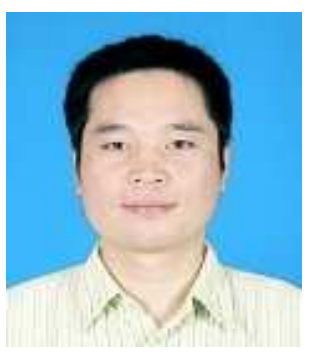

Zhengwei Chang received the Ph.D. degree in Computer Science and Technology from University of Electronic Science and Technology of China in 2009. His research interests include dependable and energy efficient embedded system, and intelligent algorithms. 